\title{
MicroRNA targeting energy metabolism in ovarian cancer: a potent contender for future therapeutics
}

\author{
Siddhika Pareek ${ }^{1}$, Aritro Nath ${ }^{2}$, R. Stephanie Huang ${ }^{1}$ \\ ${ }^{1}$ Department of Experimental and Clinical Pharmacology, University of Minnesota, Minneapolis, MN, USA; ${ }^{2}$ Department of Medical Oncology and \\ Therapeutics Research, City of Hope, Monrovia, CA, USA \\ Correspondence to: Stephanie Huang. Department of Experimental and Clinical Pharmacology, University of Minnesota, Minneapolis, MN 55455, \\ USA. Email: rshuang@umn.edu. \\ Provenance: This is an invited article commissioned by the Section Editor Dr. Xiao Li, MD (Department of Urology, Jiangsu Cancer Hospital \& \\ Jiangsu Institute of Cancer Research \& Nanjing Medical University Affiliated Cancer Hospital, Nanjing, China). \\ Comment on: Muys BR, Sousa JF, Plaça JR, et al. miR-450a Acts as a Tumor Suppressor in Ovarian Cancer by Regulating Energy Metabolism. Cancer \\ Res 2019;79:3294-305.
}

Submitted Oct 16, 2019. Accepted for publication Oct 31, 2019.

doi: $10.21037 /$ atm.2019.11.15

View this article at: http://dx.doi.org/10.21037/atm.2019.11.15

The metabolic activities of cancer cells are frequently altered to sustain their malignant properties under the influence of oncogenes and tumor suppressors (1). Therefore, it is highly pertinent to determine the key regulators of metabolic reprogramming during tumor progression. MicroRNAs (miRNAs) are one such class of small non-coding RNA regulators that influence cellular processes during both physiological and pathological conditions. In recent years, the roles of miRNAs in association with energy metabolism have gained much interest in the field of cancer.

In this league, the study published in Cancer Research by Muys et al. (2) has demonstrated that miR-450a, which is frequently downregulated in ovarian cancer, serves as a tumor suppressor by modulating energy metabolism and pathways related to metastasis. The study showcased the anti-tumorigenic and anti-metastatic effect of miR-450a in vitro using ovarian cancer cell lines (A2780 and SKOV3), and in vivo using xenograft mice models. Overexpression of mir-450a showed a significant decrease in expression levels of several important genes associated with epithelial to mesenchymal transition (EMT), an important pathway involved in the metastatic progression of cancer cells (3). For instance, the authors demonstrated a loss of vimentin (VIM), a pro-EMT marker, along with reduced migration and invasion rates of A2780 cells overexpressing miR-450a.

The authors reaffirmed their observation in vivo using miR-450a overexpressing A2780 xenografts in immunocompromised mice and found diminished tumors sizes. However, cell line-based xenograft models have few caveats including their inability to capture tumor biology, heterogeneity and host immune responses, essentially governed by the dysregulated metabolic state of cancer cells. Therefore, it might be worthwhile to extend this study in syngeneic mouse models or patient-derived xenografts (PDXs) models. Especially, PDX models distinctly recapitulate features of ovarian cancer like metastasis and ascites formation which will allow for much comprehensive studies and "omics-driven" precision medicine (4). It is noteworthy that authors also included miR-450b, a member of the same miRNA family, which expectedly showed similar tumor suppressive properties.

To explore the molecular events regulating the phenotypes induced by miR-450a overexpression, the author's further characterized the miRNA 'targetome' using AGO-PAR-CLIP in combination with RNA-Seq, a powerful high-throughput method that enables the identification of miRNA binding sites. Of the several miR-450a targets captured in this analysis, the study highlighted a number of promising energy metabolism pathway genes such as ACO2, TIMMDC1, ATP5B and MT-ND2, in addition to VIM. The ability of miR-450 to inhibit these genes, crucial for energy metabolism via the citric acid cycle and oxidative phosphorylation, might explain how miR-450 overexpression reduced tumor size and metastasis. The study further 
compared their findings to the expression levels observed in ovarian cancer and normal tissues from the Cancer Genomic Atlas database. Through this analysis, the authors reaffirmed patterns of negative correlation between ACO2 and ATP5B with miR-450a expression levels.

Metabolically malleable, cancer cells have been shown to utilize both glucose and glutamine for their growth; however, researcher have been long striving to delineate the association between the aggressiveness of cancer cells and metabolic dependencies. While cancer cells preferentially deploy aerobic glycolysis, utilizing both glucose and glutamine, their mitochondrial oxidative phosphorylation increasingly rely on glutamine metabolism, providing citric acid cycle intermediates $(5,6)$. Muys et al. (2) observed a decrease in oxidative phosphorylation and an increase in glucose uptake by nearly $80 \%$ upon miR-450a overexpression. Additionally, glutamine starvation of miR450 overexpressing A2780 cells increased the viability of the cells and reduced apoptosis. The authors suggest that is a likely outcome of miR-450a targeting ACO2, which is part of the citric acid cycle, thus altering cellular glutamine metabolism. Based on these findings authors propose that mir-450a overexpression targets the genes involved in the oxidative phosphorylation and glutaminolysis thereby resulting in increased consumption of glucose. This shift in metabolic tendencies potentially results in reduced tumorigenicity and render cancer cells less aggressive. However, these claims are partly run counter to previous findings in human (7) and mice (8) studies that show that increased glycolysis is associated with poorer survival and development and progression of ovarian cancer, respectively. While inhibition of glutaminolysis in ovarian cancer cell lines has been shown to induce apoptosis and cell cycle arrest in ovarian cancer cells through AMPK/ mTOR/S6 pathway $(9,10)$. Therefore, it seems likely that both glycolysis and glutaminolysis are crucial for ovarian cancer pathogenesis (11).

Taken together, this study is a commendable effort to establish the role of miRNAs in regulating mitochondrial energy metabolism in ovarian cancer. Future studies can benefit from the outcomes of this study and investigate the potential of miR-450a, in combination with other regulators, as targets for controlling the switch of cancer cells to alternative energy sources.

\section{Acknowledgments}

None.

\section{Footnote}

Conflicts of Interest: The authors have no conflicts of interest to declare.

Ethical Statement: The authors are accountable for all aspects of the work in ensuring that questions related to the accuracy or integrity of any part of the work are appropriately investigated and resolved.

\section{References}

1. Cairns RA, Harris IS, Mak TW. Regulation of cancer cell metabolism. Nat Rev Cancer 2011;11:85-95.

2. Muys BR, Sousa JF, Plaça JR, et al. miR-450a Acts as a Tumor Suppressor in Ovarian Cancer by Regulating Energy Metabolism. Cancer Res 2019;79:3294-305.

3. Brabletz T, Kalluri R, Nieto MA, et al. EMT in cancer. Nat Rev Cancer 2018;18:128-34.

4. Bobbs AS, Cole JM, Cowden Dahl KD. Emerging and Evolving Ovarian Cancer Animal Models. Cancer Growth Metastasis 2015;8:29-36.

5. DeBerardinis RJ, Chandel NS. Fundamentals of cancer metabolism. Sci Adv 2016;2:e1600200.

6. Yang L, Moss T, Mangala LS, et al. Metabolic shifts toward glutamine regulate tumor growth, invasion and bioenergetics in ovarian cancer. Mol Syst Biol 2014;10:728.

7. Bakhru A, Buckanovich RJ, Griggs JJ. The impact of diabetes on survival in women with ovarian cancer. Gynecol Oncol 2011;121:106-11.

8. Anderson AS, Roberts PC, Frisard MI, et al. Metabolic changes during ovarian cancer progression as targets for sphingosine treatment. Exp Cell Res 2013;319:1431-42.

9. Yuan L, Sheng X, Clark LH, et al. Glutaminase inhibitor compound 968 inhibits cell proliferation and sensitizes paclitaxel in ovarian cancer. Am J Transl Res 2016;8:4265-77.

10. Yuan L, Sheng X, Willson AK, et al. Glutamine promotes ovarian cancer cell proliferation through the mTOR/S6 pathway. Endocr Relat Cancer 2015;22:577-91.

11. Sun L, Yin Y, Clark LH, et al. Dual inhibition of glycolysis and glutaminolysis as a therapeutic strategy in the treatment of ovarian cancer. Oncotarget 2017;8:63551-61.

Cite this article as: Pareek S, Nath A, Huang RS. MicroRNA targeting energy metabolism in ovarian cancer: a potent contender for future therapeutics. Ann Transl Med 2019;7(Suppl 8):S299. doi: 10.21037/atm.2019.11.15 